\title{
Long-Term Surface Electrode Impedance Recordings Associated with Gliosis for a Closed-Loop Neurostimulation Device
}

\author{
Karl A. Sillay ${ }^{\mathrm{a}-\mathrm{c}}$ Solomon Ondoma ${ }^{\mathrm{a}}$ Brett Wingeier ${ }^{\mathrm{d}}$ Dominic Schomberg ${ }^{\mathrm{a}}$ \\ Priyanka Sharma $^{a}$ Rahul Kumar $^{\mathrm{e}}{\text { Gurwattan S. Miranpuria }{ }^{\mathrm{a}} \text { Justin Williams }}^{\mathrm{b}}$ \\ a Department of Neurosurgery, University of Wisconsin-Madison, Madison, WI, USA; ${ }^{b}$ Department of Biomedical \\ Engineering, University of Wisconsin-Madison, Madison, WI, USA; 'Department of Anatomy and Neurobiology, \\ University of Tennessee Health Science Center, Memphis, TN, USA; ${ }^{d}$ Halo Neuroscience, San Francisco, CA, USA; \\ e College of Medicine, University of Tennessee Health Sciences Center, Memphis, TN, USA
}

\section{Keywords}

Neuromodulation · Impedance · Gliosis · Electrode

\begin{abstract}
Background: Closed-loop neurostimulation is a novel alternative therapy for medically intractable focal epilepsy for patients who are not candidates for surgical resection of a seizure focus. Electrodes for this system can be implanted either within the brain parenchyma or in the subdural space. The electrodes then serve the dual role of detecting seizures and delivering an electrical signal aimed at aborting seizure activity. The Responsive Neurostimulation (RNS ${ }^{\circledR}$ ) system (Neuropace, Mountain View, CA, USA) is an FDA-approved implantable device designed for this purpose. Objective: One of the challenges of the brain machine interface devices is the potential for implanted neurostimulator devices to induce progressive gliosis, apart from that associated with the minimal trauma at implantation. Gliosis has the potential to alter impedances over time, thereby affecting the clinical efficacy of these devices, and also poses a challenge to the prospects of in vivo repositioning of depth electrodes. We present a clinical case with 3-year follow-up and pathology. Methods: Single-case, retrospective review within a ran-
\end{abstract}

\section{KARGER}

(c) 2019 S.Karger AG, Basel

E-Mail karger@karger.com

www.karger.com/aon domized trial with specific minimum follow-up and impedance measurements. Results: Impedance changes in the surface electrode over time were observed. Surgical pathological findings revealed significant gliosis in the leptomeninges of the cortices. Conclusion: We report, for the first time, longterm impedance recordings from a surface electrode associated with pathologic findings of gliosis at the Neuropace device-tissue interface in a patient who was enrolled in the multicenter RNS System Pivotal Clinical Investigation. Further study is required to elucidate the temporal relationship of pathological findings over time. Impedance changes were more complex than can be explained by a progressive or transient pathological mechanism. Further effort is required to elucidate the relationship between impedance change and seizure event capture.

(c) 2019 S.Karger AG, Basel

\section{Introduction}

Epilepsy is a medical condition that produces seizures affecting a variety of mental and physical functions. When a person has 2 or more unprovoked seizures, he or she is considered to have epilepsy. Electrical interfacing through 


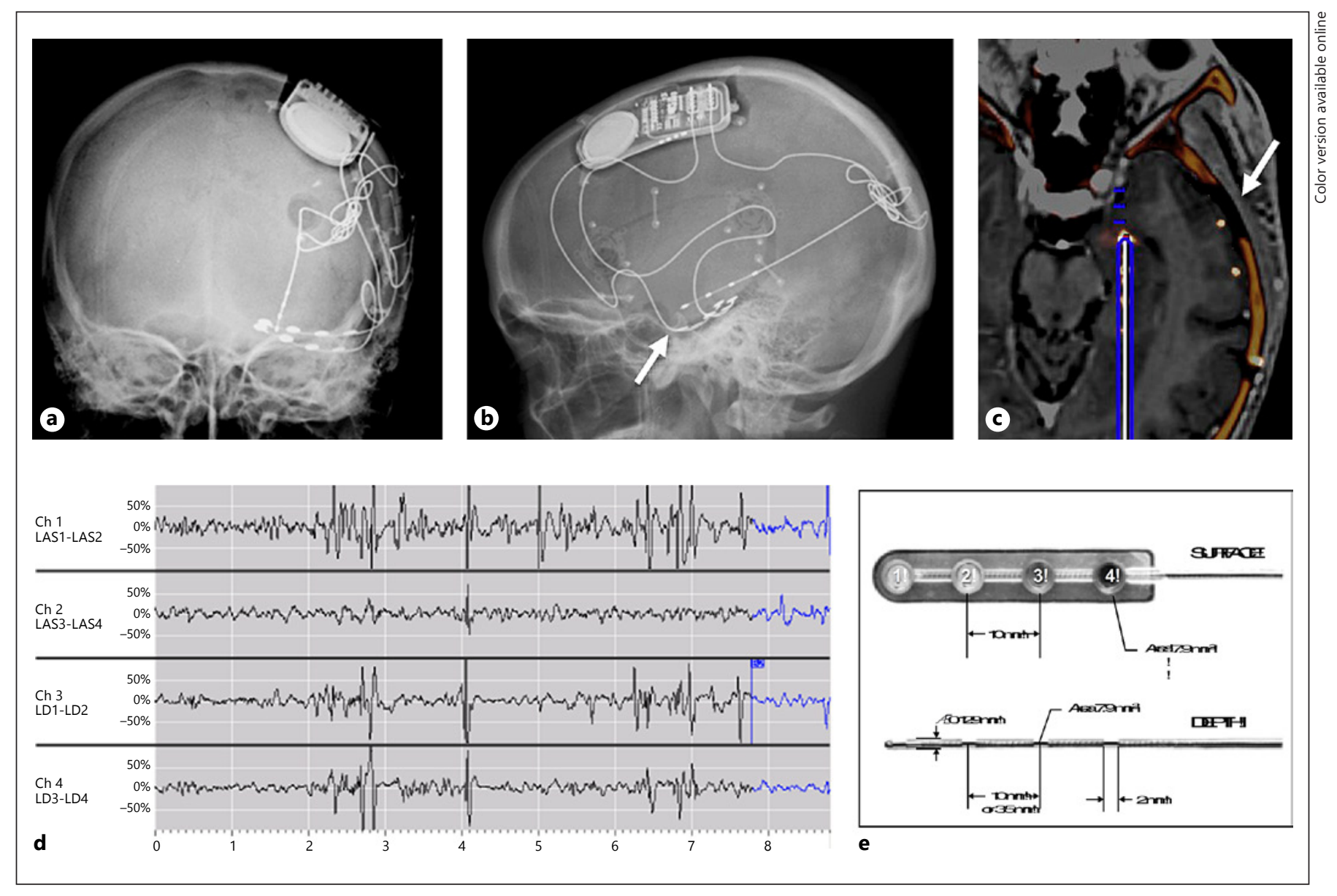

Fig. 1. Electrode location and telemetry. Surface electrode anteroposterior radiograph (a), lateral radiograph (b), postoperative CT/preoperative MRI fusion image (c), radiotelemetry (d), electrode morphology (e). Arrow marks indicate the P1 surface electrode.

the use of either microelectrodes or macroelectrodes shows promise for the treatment of many neurological conditions, including epilepsy. While neuromodulation or deep brain stimulation (DBS) has shown benefit in the treatment of movement disorders [1], systems capable of sensing and delivering a stimulus or treatment are attractive, particularly in the treatment of seizure episodes localized to eloquent cortex.

Closed-loop neurostimulation provides an attractive treatment strategy for medically intractable focal epilepsy for patients who are not candidates for surgical resection of a seizure focus or do not benefit from drug therapy [2]. Electrodes for this system are implanted either within the brain parenchyma or cortically depending upon the neuromodulation target. The electrodes then serve the dual role of detecting seizures and delivering an electrical signal aimed at aborting seizure activity. The Responsive
Neurostimulation System (RNS ${ }^{\circledR}$, Neuropace, Mountain View, CA, USA) is an investigational device under FDA review designed for this purpose. This system consists of a cranially implanted neurostimulator and leads, a physician programmer that communicates wirelessly with the implanted device, a remote monitor used by the patient to upload recorded data, and a Patient Data Management System that receives and provides access to these data. The $\mathrm{RNS}^{\circledR}$ System includes depth leads that are similar in geometry and construction to those commercially available for DBS, and subdural leads that are similar to those commercially available for intracranial monitoring (Fig. 1).

Leads are implanted to place electrodes as close as possible to the epileptic focus or foci, and the leads are routed to the neurostimulator through burr holes or a craniectomy. The RNS ${ }^{\circledR}$ leads are connected to a separately implanted neurostimulator, although future systems may 
incorporate computing technology closer to the neural interface [3]. The RNS ${ }^{\circledR}$ leads enable detection and stimulation through the same electrodes during an epileptic event. While only 2 leads can be connected to the neurostimulator at one time, additional leads were implanted in certain cases during the $\mathrm{RNS}^{\circledR}$ pivotal clinical trial to allow the inclusion of an alternate neuromodulation target. This allowed lead connections to be subsequently changed in certain cases.

The distal end of each lead bears 4 platinum/iridium electrodes of surface area $7.9 \mathrm{~mm}^{2}$ apiece. The neurostimulator continuously analyzes incoming electrocorticographic data and is programmed to deliver electrical stimulation when the patient's characteristic epileptiform activity is detected. In addition to its detection and stimulation capabilities, the neurostimulator can be configured to record up to 4 channels of intracranial EEG in response to electrographic events detected by the neurostimulator, patient triggering of recording by swiping a magnet near the neurostimulator, or according to a predefined schedule. All data are subjected to low pass filtering before digitization at a sampling rate of $250 \mathrm{~Hz}$.

Impedance measurements are considered to be a marker to test the efficacy of implanted electrodes for many reasons. Measurements of electrical impedance use the same interface for recording and for stimulation. Changes in impedance measurements can indicate a problem with the electrode or electrode tissue interface. For example, a decrease in impedance could detect a hardware malfunction caused by a short circuit or breakdown of electrode insulation or hardware failure causing an open circuit. Changes in impedance have been reported to vary with time since implantation, and may provide insight into device tissue physical changes over time [1]. Finally, the use of impedance values has been proposed to support the development of surrogate gel or phantom brain models used to develop a baseline impedance value in-vitro as modeling observed in-vivo and observed human properties of brain tissue such as impedance and capacitance [4].

One of the challenges of the brain machine interface devices is the potential for implanted neurostimulator devices to induce progressive gliosis, apart from that associated with the minimal trauma at implantation. The gliosis in turn has the potential to alter impedances over time, thereby affecting the clinical efficacy of these devices and also posing a challenge to the prospects of in vivo repositioning of depth electrodes. Impedance measurements are higher with a more reactive tissue or with a more pathological tissue. These changes in tissue cause a problem in microelectrodes as they decrease signal amplitude over time. Accurate electrographic readings are a requirement of neurostimulator devices and this data is dependent upon the interaction between the electrode and the tissue - specifically, the amount of activated tissue between the electrodes, the properties of the surrounding tissue, impedance of the electrode-tissue interface, and the design of the depth and subdural electrodes [1].

\section{Electrode Locations and Telemetry \\ Patient History}

The patient was a 30 -year-old, right-handed woman who was diagnosed with partial complex epilepsy at age 4. Her seizures often generalized and became intractable to 4 antiseizure medications. Her first consultation for possible surgical resection was in 2007. At this point she was having weekly seizures. Her neurologic exam and at least 2 brain MRIs were essentially normal. Routine interictal EEG revealed diffuse slowing with superimposed bursts of high voltage generalized delta with bifrontal prominence. fMRI showed moderate left language dominance. PET scan revealed left temporal hypometabolism. Unfortunately, the patient did not tolerate WADA testing. During invasive monitoring, 3 typical complex partial seizures with secondary generalization were captured, one of which was well localized to the left mesiotemporal region. Because of her left language dominance, she was not considered a good surgical candidate at that point. After presentation to and review of the multidisciplinary epilepsy conference, the patient was recommended for inclusion in the $\mathrm{RNS}^{\circledR}$ the multicenter RNS System Pivotal Clinical Trial. In October 2007, a stereotactically implanted left temporal depth electrode, 2 subtemporal strip electrodes, and the Neuropace RNS device were implanted. The patient was eventually randomized to the treatment arm of the trial. However, after a period of reduced seizure activity, the patient presented with focal status epileptics in 2008 and seizures remained poorly controlled thereafter. Surgical resection was reconsidered and in 2010 the patient underwent a temporal lobectomy with en bloc removal of the electrodes with the attached brain tissue, which was sent for neuropathologic analysis. Seizure control was achieved after surgical resection.

\section{Methods}

Impedance Measurements

Impedance data were collected at prospectively designed intervals as well as around the time of device programming. Impedance measurements were also collected during routine patient visits in 
the context of a multicenter, double-blind, randomized controlled trial of responsive neurostimulation for medically intractable partial onset epilepsy, and a subsequent open-label long term treatment trial following the same group of patients. Patient visits were scheduled at 2, 4, 5, 6, 7, and 8 weeks post implant; at 4-week intervals from 8 to 56 weeks post implant; at 12-week intervals from 56 to 104 weeks; and at 6-month intervals thereafter. Because the actual timing of patient visits and thus the timing of impedance measurements varied according to clinical needs, impedance data are reported here with the actual elapsed times post implant. The data reported here also include data collected at unscheduled follow-up visits, and multiple measurements collected within the same visit.

Impedance was determined for each electrode individually by the RNS ${ }^{\circledR}$ System using voltage measurements collected during delivery of single current-controlled monopolar pulses (i.e., using the neurostimulator case as the return electrode). Pulses used for impedance measurement were charge-balanced; biphasic square wave pulses delivered the anodic phase first at $3 \mathrm{~mA}$ amplitude and $40 \mu$ s pulse width per phase.

\section{Surgical Procedure and Removal of Tissue}

Detailed in Appendix A.

\section{Pathological Analysis}

Specimens were sent for pathological analysis as below:

NeuroPace Depth Electrode

Lateral Temporal Cortex

Deep Temporal White Matter

Anterior Subtemporal Electrode with Attached Brain

Amygdala

Posterior Subtemporal Electrode with Associated Brain

Hippocampus Microscopic Analysis

Twelve H\&E-stained slides were examined. Also, some specimens were stained with LFB-PAS and Bielschowsky silver. Others were immunolabeled with NeuN and GFAP antibodies.

\section{Results}

No significant major morbidity was attributable to chronic surface electrode implantation. Chronic changes associated with implantation were identified.

\section{Impedance}

Impedance data were collected over 730 days. The mean impedance was $938 \Omega$ with a SD of $251 \Omega$ for all 4 electrodes (Fig. 2a). There was a trend of impedance change over time. A best-fit curve demonstrates a slope of $0.45 \Omega$ /day and was statistically significantly different from zero (Fig. 2b).

\section{Pathologic Diagnosis}

Left Temporal Lobe Partial Lobectomy and Removal of NeuroPace Device:
1) Meningeal fibrosis with mild chronic inflammation as well as focal subjacent cortical reactive changes noticed over the posterior subtemporal electrode with associated brain.

2) Leptomeningeal fibrosis with mild chronic inflammation, anterior subtemporal electrode with subjacent cortex.

3) Mild subpial gliosis as well as possible mild white matter gliosis, noted in the lateral temporal lobe and deep white matter.

\section{Pathology}

The most significant morphologic findings in these partial temporal lobectomy specimens lie in the leptomeninges of the cortices associated with electrode placement. The anterior specimen is less involved than the posterior specimen, which reveals some cortical degenerative changes as well as leptomeningeal fibrosis. There may be a slight white matter gliosis subjacent to this material as is also seen in the sample of deep white matter (specimen 3). In the lateral temporal lobe, there was only mild subpial gliosis with mild corpora amylacea in combination with moderate corpora amylacea in the deeper white matter. The hippocampal sections were somewhat fragmented and precluded complete assessment of the cornu ammonis. None of the sections revealed any evidence of encephalitis, vasculitis, cerebral cortical dysplasias, hamartomas, or neoplasias. There were no significant numbers of heterotopic neurons seen in the white matter. Gliosis associated with closed loop neurostimulator device is shown in Figure 3a-c.

\section{Discussion}

The investigation is limited by the availability of tissue certain to be found around the implanted devices. As the electrode-tissue interface is critical to neural recording and neurostimulation performance, analysis of measurable properties such as brain impedance is essential to understanding the dynamics of the electrode-tissue interface. The current study details longitudinal impedance measurements of both depth and subdural electrodes in a large cohort of patients in a multicenter clinical trial of responsive neurostimulation for epilepsy. Both subdural and depth electrodes have demonstrated efficacy in terms of long-term impedance stability, which suggests that EEG readings will be accurate. Knowledge of impedance values is useful to quantify the relationship between an implanted electrode and brain tissue as voltage is applied. 


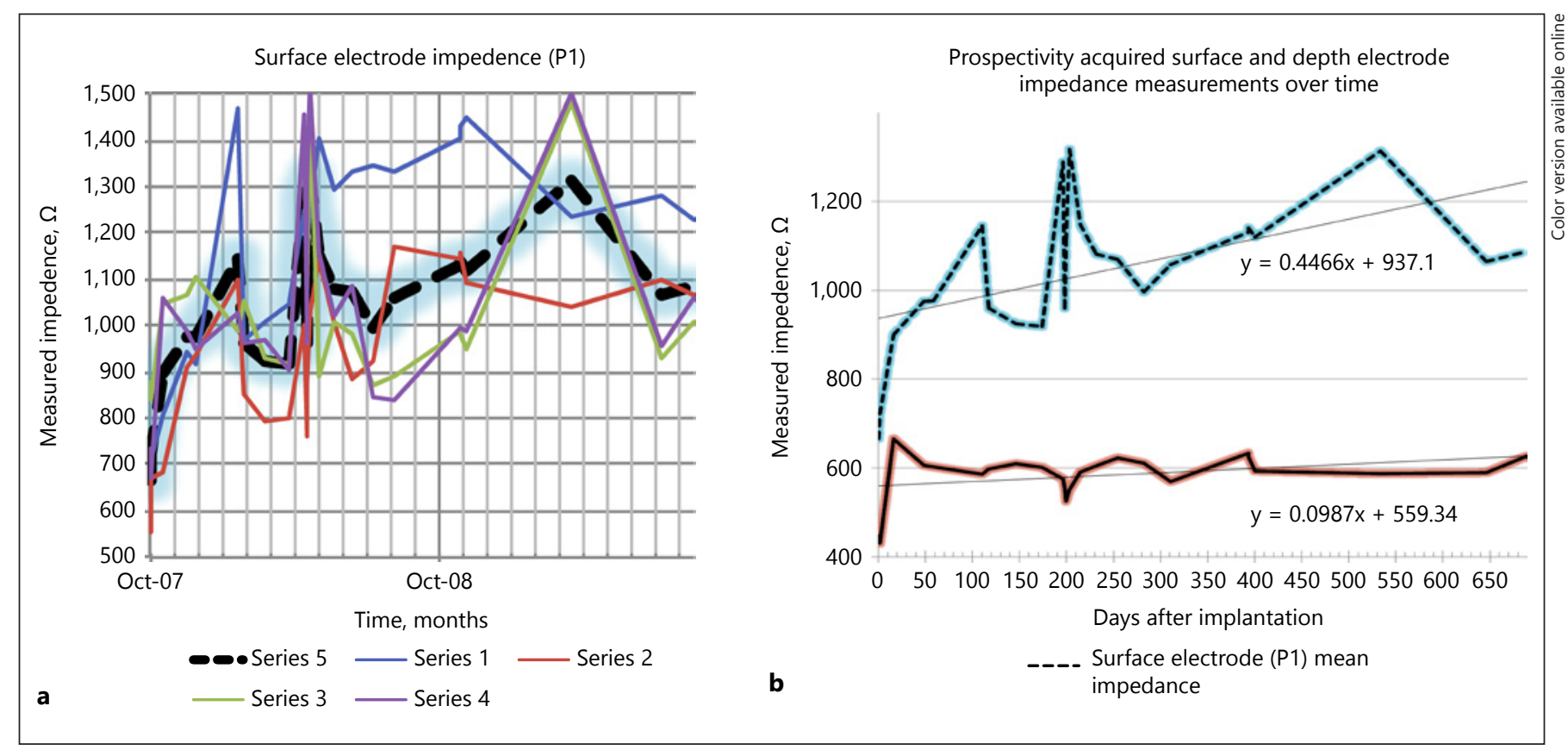

Fig. 2. a Surface electrode impedance over time. Series 1, 2, 3, and 4 represent the respective surface electrode contacts. Series 5 represents the mean impedance. $\mathbf{b}$ Prospectively acquired surface and depth electrode impedance measurements over time. Surface elec-

trode (above, dashed line, P1, indicated in Fig. 1) mean impedance vs. depth electrode mean impedance (below, continuous line) over time.

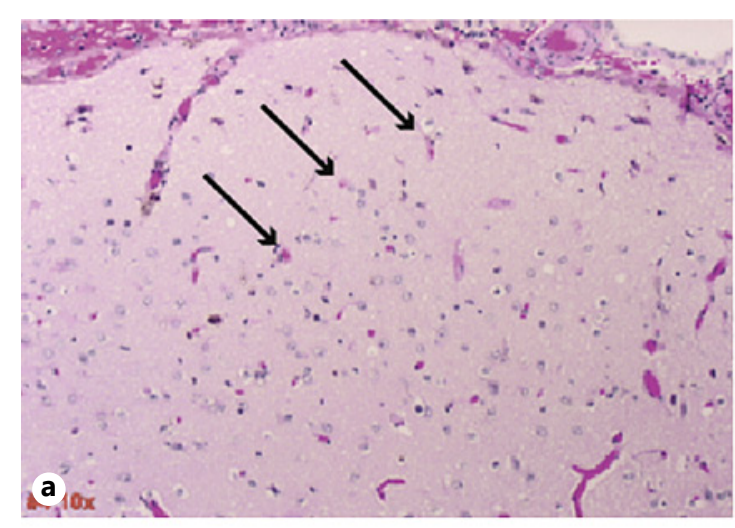

Fig. 3. Gliosis associated with a closed loop neurostimulator device. H\&E and GFAP stained sections of subtemporal lobe cortical tissue attached to depth electrodes. Arrows show reactive astrocytes. a 10X H\&E tissue section of the temporal lobe cortex previously attached to an anterior sub-temporal electrode. b, 10X GFAP stained tissue section as described in a. c, 10X GFAP section of temporal lobe cortex previously attached to a posterior sub-temporal electrode showing reactive astrocytes and gliosis.
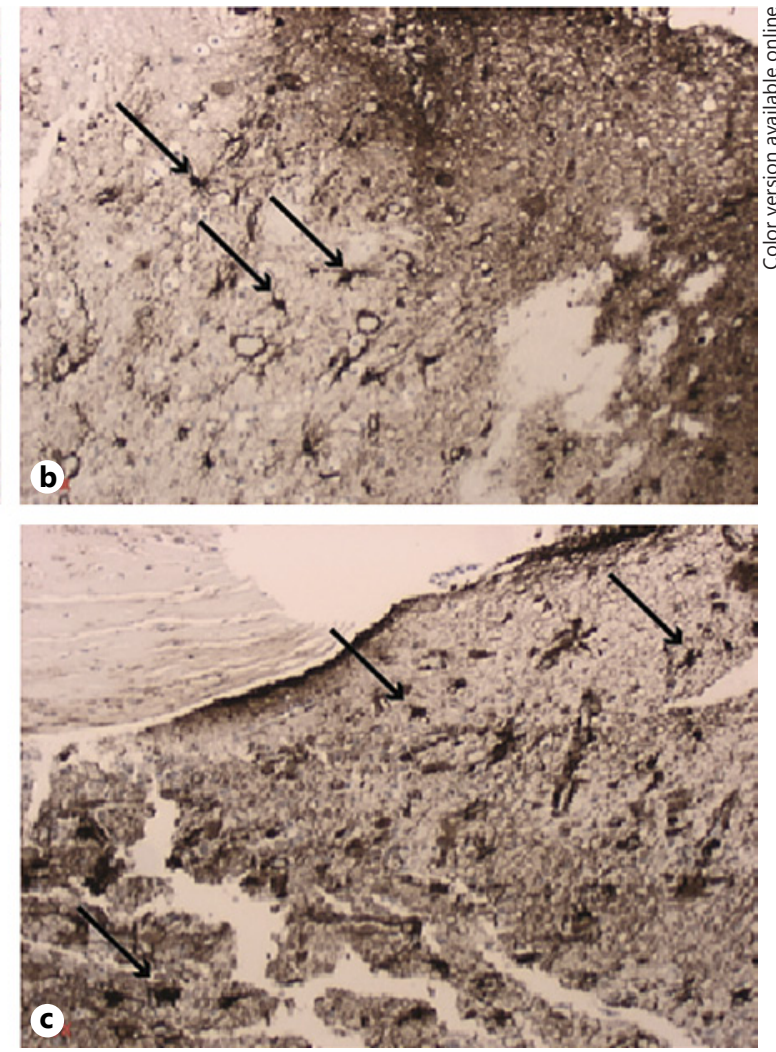
This is further supported by agarose gel models used to develop a baseline impedance value in vitro as they possess in vivo properties of brain tissue such as impedance and capacitance.

1. Accuracy of measurements and long-term stability: The ability of an implanted neurostimulator to provide an accurate assessment of impedance is the first step in interrogating the electrode-tissue interface as is the case for the RNS device [1].

2. Dynamics of the peri-implant period: Prior to the current RNS device based impedance/pathology correlate report, the tissue response to implanted electrodes has been studied extensively. Both reactive tissue and consolidated scar tissue exhibit higher resistive properties than the normal tissue [5-10]. Pre-clinical, peri-implant impedance change has been reported in chronically implanted microelectrodes, consistent with tissue remodeling at the electrode-tissue interface with a time course similar to that of the general reactive gliosis response [5, 10-13]. Previous attempts at characterizing the peri-implant period using available neurostimulators and standard impedance measuring protocols often resulted in impedances exceeding device measurement capabilities during the perioperative period. Furthermore, previous measurements of impedance associated with therapeutic stimulation pulses allowed direct comparison of impedance value only insofar as stimulation settings remained constant. Finally, due to surgical factors such as implant staging, implantable pulse generators capable of measuring impedance were not generally implanted or used for impedance measurements until after leads were implanted and until much of the hypothesized period of dynamic changes would have elapsed $[14,15]$.

Previous publications have reported transient increases in impedance in depth and subdural electrodes followed by a decrease after the acute phase of the tissue reaction has passed in animals and in a large clinical trial $[1,6,10,12,16,17]$. We have previously reported "... a trend for impedance to increase over the first 12 weeks after implant. Impedance then stabilized and remained stable at 1 year after implant. This is consistent with progressive change of the subdural electrode-tissue interface, which is initially separated by a small layer of cerebrospinal fluid and then progressively adheres." Similar time courses of impedance change are reported elsewhere in the literature [18]. Neuropathological examination of the implantation site of subdural electrodes implanted for diagnostic epilepsy procedures has shown short-term changes associated with tissue reaction [19]. Long-term changes have been confirmed in this patient population, including one re-operated patient with the removal of an implanted electrode at approximately 2 years after the operation. The electrode firmly adhered to the pial surface of the temporal lobe, requiring sharp dissection for removal (Sillay, personal obervation).

Electrode impedance may be influenced by physical characteristics, such as electrode configuration and surface area (16, Fig. 1e), as well as dynamic and reactive processes (Fig. 2). Thickness and conductivity of the encapsulation layer around the electrode, bulk tissue conductivity, and electrical stimulation activity itself contribute significantly to electrode impedance [20-22]. The time course of the transient changes in impedance may be related to microglial activation and subsequent fusion into multinuclear giant cells or mild to moderate inflammation as a potential mechanism of action for peri-implant impedance change with resulting GFAP positive pathology findings [23, 24] (Fig. 3). Collagen production has been shown to increase in the dura and subarachnoid space following brain surface injury, which has the potential to produce a fibrotic response [25]. A more fibrotic response would typically be acellular in nature, which would theoretically have a different impedance signature from that of the highly cellularized astrogliotic response that has been typically characterized around penetrating devices $[13,26]$.

\section{Neurostimulation and Impedance Change}

As previously reported, no difference was noted in impedance in those randomized to sham stimulation in unblinded patients at 5 months post-implant [1] or to actual stimulation. Impedance stability and peri-implant changes were not different between the non-stimulated and stimulated groups and were not different in patients who received no test stimulations through the fourth week post-implant [1]. Electrical stimulation has been shown in animal models to alter measured electrode-tissue impedance in microelectrodes. Short DC voltage pulses have been reported to decrease overall microelectrode impedance and subsequently increase recorded neural signal amplitude [27]. These effects are reversible, as impedance gradually returns to pre-stimulation levels. The component that changes most significantly is related to tissue impedance, suggesting that electrical stimulation can alter the biophysical properties of the brain parenchyma directly surrounding the implanted electrode site. The impedance of more macro-sized devices has also been shown to change dramatically post implantation during clinically relevant neurostimulation $[12,28]$. For example, using a scaled down DBS electrode in a primate 
model electrical stimulation resulted in a significant but reversible decrease in impedance magnitude. Electrical impedance changes as a result of electrical stimulation have also been documented in humans with cochlear implants $[29,30]$.

\section{Clinical Correlations of Electrode-Tissue Impedance}

Further investigations can determine whether there is a link between impedance and therapeutic effect. Understanding the mechanisms underlying impedance change may lead to strategies to alter tissue reactions and ultimately provide a stable environment for the electrodetissue interface or even to new strategies for optimization of therapeutic stimulation.

Little has been published regarding the magnitude and variability of impedance of stimulation paths used therapeutically in vivo, although there is a general sense that initial post-operative DBS programming should follow a period of recovery from postoperative changes and that electrode impedance may fluctuate during this period. Clinically, the focus of impedance measurements has been to ensure tissue safety and diagnose system malfunction such as electrode discontinuity [31-34].

A broader understanding is emerging in the clinical relevance of impedance effects to voltage-controlled stimulation. Regarding current delivery, tissue activation volume, and stimulation thresholds, the effect of voltagecontrolled stimulation is dependent on the electrode-tissue interface impedance $[9,20,29,35,36]$. In one example of impedance changes that might affect voltage-controlled stimulation, transient unusually high electrode impedance has been documented in DBS electrodes reactivated after a period of time, which subsequently normalized spontaneously [32]. Neither of these findings was identified in the impedance data reported here.

Taking mean surface electrode impedances as an example, a change was observed from $721 \Omega$ within the first week of implantation rising to $1,146 \Omega$ by week 16 and then falling to $919 \Omega$ by week 24 . Considering the worst-case scenario by using $\pm 250 \Omega$ as values for the $95 \% \mathrm{CI}$, these changes could be as dramatic as $471 \Omega$ to $1,396 \Omega$ to $669 \Omega$, respectively. Under voltage-controlled stimulation, if programmed at the nadir of the first week, neurostimulation current would decrease by $35 \%$ by week 104 . If programming at the peak of 1,288 $\Omega$, actual neurostimulation current would increase by $23 \%$ within 12 weeks of programming. Given these dynamics, the current controlled stimulation design appears best positioned to provide delivered neuromodulation stability.

Long-Term Surface Electrode Impedance with Gliosis

\section{Conclusion}

Measurement capabilities provided by clinical and investigational neurostimulation devices have expanded from the ability to detect system discontinuity to the ability to detect (when averaged over a large cohort) separate electrical signatures for likely divergent peri-implant changes at the electrode-tissue interface. Further investigation is warranted to elucidate the mechanism of action of impedance change based upon implant tissue environment. Future analysis and new technologies may allow sensing and stimulating systems employing electrode-tissue interface coupling to reveal clinically meaningful interpretations of the relationship of impedance change to the implant tissue environment, continuity and effectiveness of neurostimulation, and stimulation parameters.

Understanding these reactive processes may provide information that could contribute to the design characteristics of future electrodes and therefore further study is warranted. Understanding these dynamic changes in the electrode-tissue interface could also explain, at least in part, why the clinical response to implanted electrodes changes over time. Further, elucidation of tissue-level responses to implanted electrodes, such as those produced by microglia at the interface, could be used to develop strategies to prevent these impedance changes from occurring [37]. The time course of these impedance changes is consistent with the time course of a transient favorable effect of electrode implantation that is described in studies of neurostimulation for epilepsy in persons who are not receiving stimulation and to the time course of the clinical response to DBS in Parkinson's disease and various forms of dystonia $[16,38]$.

\section{Acknowledgments}

The authors are thankful to Dr. M. Shahriar Salamat, Department of Pathology and Laboratory Medicine (Neuropathology), VA Hospital, Madison-Wisconsin for his help in pathologic diagnosis. The constant guidance received from Dr. Robert J Dempsey, Chairman, Department of Neurological Surgery is gratefully acknowledged. The study was supported by internal funds from the department of Neurological Surgery, University of Wisconsin, School of Medicine and Public Health.

\section{Disclosure Statement}

The authors declare that they have no competing interests to disclose and this manuscript complies with ICMJE.

Ann Neurosci 2018;25:289-298

DOI: $10.1159 / 000481805$ 


\section{Author Contribution}

The study was designed by K.A.S. and performed by S.O., B.W., and J.W. The manuscript was written by K.A.S., G.S.M., D.S., P.S., and R.K. Pathological analysis of specimens was performed by Dr. M. Shahriar Salamat.

\section{Appendix A}

Surgical procedure and removal of tissue:

\section{A. Placement of NeuroPace device 10/10/2007}

Having obtained informed and written consent, the patient was taken to the OR and administered general anesthesia with endotracheal intubation. Under sterile conditions, a CRW frame localizer was placed on the patient's head. Thereafter, the patient was transferred to the MRI suite with the CRW frame localizer in place for preoperative localization image acquisition. She was returned to the OR and placed in a semi-sitting position. Her left hemicranium was clipped. She was prepped and draped in a sterile fashion. Simultaneously, the images acquired using preoperative images were loaded into the Medtronic Stealth workstation using the FrameLink program for trajectory planting for the left epicanthal depth electrode. Final target coordinates were lateral or $\mathrm{X}$ equal to -20.8 , AP or $\mathrm{Y}$ equal to +13.0 , vertical or $\mathrm{Z}$ equal to +2.1 , ring of +11.9 , and arc of 5.0 left. The stereotactic frame coordinates were set both on the stereotactic phantom and the stereotactic frame. After all coordinates were crosschecked, the CRW stereotactic arc was affixed to the CRW stereotactic frame containing the navigation information based on the previously obtained MRI scan.

A preoperative skin trajectory mark was made on the left occipital area. A 1/4 arc incision with 1 end based posteriorly and 1 end based laterally was marked such that hardware would be hidden underneath the skin and the burr hole would be some distance from the skin edge. A lidocaine Marcaine mixture of anesthetic with epinephrine was infiltrated. A skin incision was made to the skull. Again, the trajectory was marked using the stereotactic frame, this time on the skull. A high-speed perforating 14-mm drill bit was used to create the burr hole. Thereafter, bone wax was applied to the bone edges and the Navigus cap affixed in the standard fashion.

At this point, the Radionics Lesion Generator probe was measured to a distance of $175 \mathrm{~mm}$, being $160 \mathrm{~mm}$ to arc center of the standard CRW frame, with an additional $10 \mathrm{~mm}$ for the cannula holder and an additional $5 \mathrm{~mm}$ for the Radionics Lesion Generator cannula. This was double checked and verified and the lesion generator probe inspected prior to use.

The dura mater was then opened in a cruciate fashion. The dural edges were cauterized. A small area of cortex was coagulated using bipolar electrocautery and a \#11 blade was used to incise the pia. After ensuring that the patient was not hypertensive, the Medtronic Lesion Generator probe was slowly advanced to its target. The NeuroPace full contact depth electrode was opened, inspected, and set at a depth of $170 \mathrm{~mm}$ with a goal of terminating 5 $\mathrm{mm}$ proximal to the distance traversed by the lesion generator probe. This was chosen to approximate the amygdalohippocampal junction.
At this point, the stereotactic lesion generator probe was withdrawn and the depth electrode passed to its target depth. Prior to passing the NeuroPace depth electrode, the lateral C-arm fluoroscopy was used to ensure that the lesion generator probe had in fact reached the frame center target. After passing the NeuroPace depth electrode to the target $-5 \mathrm{~mm}$, lateral C-arm fluoroscopy verified this position. At this point, attention was turned to backing out the lesion generator guide cannula such that the Navigus catheter could be accessed for stem lock clip placement. Again, lateral C-arm fluoroscopy was used to verify that no movement had occurred at this step. The Navigus clip was applied and lateral C-arm fluoroscopy again revealed that there had been no movement during this step. At this point, the stem lock cap was placed and C-arm fluoroscopy demonstrated that the distal contact of the NeuroPace depth electrode was indeed at the desired target of $-5 \mathrm{~mm}$.

Attention was then turned to tunneling approximately $10 \mathrm{~cm}$ of electrode anteriorly within the guide area within the plastic sleeve provided with interim pace device. Additional redundant electrode length was coiled away from the skin incision. The wound was copiously irrigated with antibiotic solution, the incision was closed in layers, and a sterile dressing applied. The patient was carefully removed from the CRW head frame. The patient was positioned with a shoulder roll under the left shoulder and placed in Mayfield pins with head rotated to the right. A standard reverse question-mark skin incision was drawn after the NeuroPace template was used on the patient's skull to approximate the implantation location. A standard temporal craniotomy was then performed.

The NeuroPace ferrule location was mapped. The perforator was again used to make a $14-\mathrm{mm}$ burr hole at this location and then the B1 Midas Rex with footplate was used to turn a craniectomy flap. Hemostasis having been achieved, the dura was opened superior to the inferior exposure designed to give access to the inferior temporal gyrus. The EEG team prepared for the electrophysiology and then anterior and posterior $1 \times 4$ contact strip electrodes were placed laterally to reach to the inferomedial aspect of the temporal lobe. Electrocorticography revealed medial spiking in the more anterior greater than more superior strip electrode. Radiographic images were taken to verify the location and orientation of the anterior-posterior location and orientation of these electrodes. Appearing at the correct anterior-posterior location and seeming to point medially, and with electrographic confirmation, the NeuroPace paddle electrodes were placed after removing the Ad-Tech disposable electrocorticography and the $1 \times 4$ strip electrodes.

The flanges were bent for the proper depth on the ferrule and the NeuroPace device was installed. The depth electrode was placed in the most lateral contact receptor and the anterior of the two $1 \times 4$ strip electrodes was placed in the more medial of the 2 receptors. The more posterior strip electrode distal contact was covered with the provided plastic sleeve and placed adjacent to the pulse generator, should it be desired that this most posterior strip be employed in the future. Telemetry of the NeuroPace device was performed.

The wound was irrigated with antibiotic solution and closed in layers. The patient was removed from the CRW stereotactic frame, extubated, and transferred to the intensive care unit.

\section{B. Removal of the Neuropace Device and Anterior temporal lobectomy 3/21/2011}

After informed consent was obtained, including a discussion of the operation, the risks, benefits, and alternatives thereto, the pa- 
tient was brought to the operating theater where general anesthesia was administered. Her hair was clipped, and her head was prepped and draped in the usual sterile fashion; after her head was affixed to the Mayfield headholder, she was positioned on the OR table with all bony prominences appropriately padded.

An incision was made through her previous posterior scar at the site of implantation of her hippocampal depth electrode. The dissection was carried down to the level of the periosteum with a combination of sharp dissection and electrocautery. The electrode was located and the burr hole through which the electrode was placed was covered with a StimLock device. The cap of the StimLock was removed, the locking mechanism was disengaged, and the depth electrode was removed. The entire length of the electrode that was visible through this incision was cut at the spot where it was tunneled anteriorly and removed, sending the tip for pathologic analysis and the wire to the company representative. The StimLock cap was then replaced, and the wound was copiously irrigated and closed in layers with Vicryl sutures in the galea and nylon suture in the skin. A sterile dressing was applied.

Another incision was made through her previous scar making a myocutaneous skin flap. The NeuroPace device, along with the electrodes, was identified. The remaining wire from the depth electrode was cut where it interfaced with the device and passed off the field. There remained 2 subtemporal electrodes, one of which, the anterior subtemporal electrode, was hooked up to the NeuroPace device, and the other, the posterior subtemporal electrode, was adjacent to the device having been placed as an alternate option for stimulation. This posterior electrode was not hooked up to the device. The anterior electrode was cut free of the device and the NeuroPace stimulator itself was removed. The prosthesis or pseudodevice was then brought into the field and placed in the NeuroPace holder. Per the manufacturer's recommendations, it was tightened in place with the locking mechanism. This wound was copiously irrigated and closed.

The anterior temporal lobectomy was then performed as described [39]. Briefly, a temporal craniotomy is performed flush with the middle fossa. The Sylvian fissure is exposed after which the posterior extent of resection is determined as $4.5 \mathrm{~cm}$ in the dominant temporal lobe and $5.5 \mathrm{~cm}$ in the nondominant one. In a step-wise fashion, the lateral neocortex is removed along with part of the fusiform gyrus, parallel to the Sylvian fissure. The temporal horn is maintained as the medial limit in the coronal plane, usually with cotton Pattie. The amygdala, uncus, fimbriae, hippocampus, and collateral eminence are identified and subpially resected along with the parahippocampal gyrus.

\section{Pathological Analysis}

Specimen "No. 1" labeled "NeuroPace Depth Electrode" consisted of an electrode measuring approximately $4 \mathrm{~cm}$ in length.

Specimen "No. 2" labeled "Lateral Temporal Neocortex" consisted of one piece of brain tissue measuring $3.5 \times 2.3 \times 0.5 \mathrm{~cm}$. The specimen was serially sectioned into 8 blocks and entirely submitted in cassettes "2A," "2B," "2C," and " $2 \mathrm{D}$."

Specimen "No. 3" labeled "Deep Temporal White Matter" consisted of 3 pieces of white to light tan tissue, ranging in size from $1.6 \times 0.6 \times 0.4$ to $0.4 \times 0.2 \times 0.2 \mathrm{~cm}$. The specimen was entirely submitted in cassette " 3 ."

Specimen "No. 4" labeled "Anterior Subtemporal Electrode with Attached Brain" consisted of a piece of brain tissue with attached electrode. The brain tissue measured $1.7 \times 1.3 \times 1.1 \mathrm{~cm}$. The electrode was attached to the edge of the specimen. After removal of the electrode, a shaved section of the brain adjacent to the electrode was placed in cassette " $4 \mathrm{~A}$ ". The rest of the brain tissue was entirely submitted in cassette " $4 \mathrm{~B}$."

Specimen "No. 5" labeled "Amygdala" consisted of one piece of white to light tan tissue measuring $0.5 \times 0.2 \times 0.2 \mathrm{~cm}$. The specimen was entirely submitted in cassette " 5 ."

Specimen "No. 6" labeled "Posterior Subtemporal Electrode with Associated Brain" consisted of a piece of brain tissue and attached electrode. The brain tissue measured $1.9 \times 1.2 \times 0.4 \mathrm{~cm}$. After shave of the electrode, a section adjacent to the electrode was submitted in cassette " $6 \mathrm{~A}$ ". The remainder of the brain tissue was entirely submitted in "6B."

Specimen "No. 7" labeled "Hippocampus" consisted of one piece of tan to dark brown tissue measuring $2 \times 1.5 \times 1.1 \mathrm{~cm}$. The specimen was further sectioned into 4 blocks and entirely submitted in cassettes "7A" and "7B."

\section{References}

1 Sillay KA, Rutecki P, Cicora K, et al: Longterm measurement of impedance in chronically implanted depth and subdural electrodes during responsive neurostimulation in humans. Brain Stimul 2013;6:718-726.

2 Guillory KS, Askin RE, Smith CF, McDonnall D, Hiatt S, Wilder AM: Wireless Electrocortigraph (ECoG) Recording System. Neural Engineering, 2011.

3 Blau A, Ziegler C, Heyer M, et al: Characterization and optimization of microelectrode arrays for in vivo nerve signal recording and stimulation. Biosens Bioelectron 1997;12: 883-892.

4 Pomfret R, Sillay K, Miranpuri G: An exploration of the electrical properties of agarose gel: characterization of concentration using nyquist plot phase angle and the implications of a more comprehensive in vitro model of the brain. Ann Neurosci 2013;20:99-107.

5 Mercanzini A, Colin P, Bensadoun JC, Bertsch A, Renaud P: In vivo electrical impedance spectroscopy of tissue reaction to microelectrode arrays. IEEE Trans Biomed Eng 2009;56:1909-1918.

6 Otto KJ, Rousche PJ, Kipke DR: Microstimulation in auditory cortex provides a substrate for detailed behaviors. Hear Res 2005;210: 112-117.

7 Schwartz AB, Cui XT, Weber DJ, Moran DW: Brain-controlled interfaces: movement restoration with neural prosthetics. Neuron 2006; 52:205-220.

8 Smith JR, Sillay K, Winkler P, King DW, Loring DW: Orbitofrontal epilepsy: electroclinical analysis of surgical cases and literature re- view. Stereotact Funct Neurosurg 2004;82: 20-25.

9 Wei XF, Grill WM: Current density distributions, field distributions and impedance analysis of segmented deep brain stimulation electrodes. J Neural Eng 2005;2:139-147.

10 Williams JC, Hippensteel JA, Dilgen J, Shain W, Kipke DR: Complex impedance spectroscopy for monitoring tissue responses to inserted neural implants. J Neural Eng 2007;4:410-423.

11 Johnson MD, Otto KJ, Kipke DR: Repeated voltage biasing improves unit recordings by reducing resistive tissue impedances. IEEE Trans Neural Syst Rehabil Eng 2005;13:160-165.

12 Lempka SF, Miocinovic S, Johnson MD, Vitek JL, McIntyre CC: In vivo impedance spectroscopy of deep brain stimulation electrodes. J Neural Eng 2009;6:046001. 
13 Turner JN, Shain W, Szarowski DH, et al: Cerebral astrocyte response to micromachined silicon implants. Exp Neurol 1999;156:3349.

14 Deuschl G, Schade-Brittinger C, Krack P, et al: A randomized trial of deep-brain stimulation for Parkinson's disease. N Engl J Med 2006;355:896-908.

15 Sillay KA, Chen JC, Montgomery EB: Longterm measurement of therapeutic electrode impedance in deep brain stimulation. Neuromodulation 2010;13:195-200.

16 Morrell MJ: Responsive cortical stimulation for the treatment of medically intractable partial epilepsy. Neurology 2011;77:12951304.

17 Satzer D, Lanctin D, Eberly LE, Abosch A: Variation in deep brain stimulation electrode impedance over years following electrode implantation. Stereotact Funct Neurosurg 2014; 92:94-102.

18 Lungu C, Malone $\mathrm{P}, \mathrm{Wu}$ T, et al: Temporal macrodynamics and microdynamics of the postoperative impedance at the tissue-electrode interface in deep brain stimulation patients. J Neurol Neurosurg Psychiatry 2014; 85:816-819.

19 Fong JS, Alexopoulos AV, Bingaman WE, Gonzalez-Martinez J, Prayson RA: Pathologic findings associated with invasive EEG monitoring for medically intractable epilepsy. Am J Clin Pathol 2012;138:506-510.

20 Butson CR, Maks CB, McIntyre CC: Sources and effects of electrode impedance during deep brain stimulation. Clin Neurophysiol 2006;117:447-454.

21 Grill WM, Mortimer JT: Electrical properties of implant encapsulation tissue. Ann Biomed Eng 1994;22:23-33.
22 Cheung T, Nuno M, Hoffman M, et al: Longitudinal impedance variability in patients with chronically implanted DBS devices. Brain Stimul 2013;6:746-751.

23 Moss J, Ryder T, Aziz TZ, Graeber MB, Bain PG: Electron microscopy of tissue adherent to explanted electrodes in dystonia and Parkinson's disease. Brain 2004;127:2755-2763.

24 Stephan CL, Kepes JJ, SantaCruz K, Wilkinson SB, Fegley B, Osorio I: Spectrum of clinical and histopathologic responses to intracranial electrodes: from multifocal aseptic meningitis to multifocal hypersensitivity-type meningovasculitis. Epilepsia 2001;42:895901.

25 Sajanti J, Bjorkstrand AS, Finnila S, Heikkinen E, Peltonen J, Majamaa K: Increase of collagen synthesis and deposition in the arachnoid and the dura following subarachnoid hemorrhage in the rat. Biochim Biophys Acta 1999;1454:209-216.

26 Polikov VS, Tresco PA, Reichert WM: Response of brain tissue to chronically implanted neural electrodes. J Neurosci Methods 2005;148:1-18.

27 Sommakia S, Rickus JL, Otto KJ: Effects of adsorbed proteins, an antifouling agent and long-duration DC voltage pulses on the impedance of silicon-based neural microelectrodes. Conf Proc IEEE Eng Med Biol Soc 2009;2009:7139-7142.

28 Wei XF, Grill WM: Impedance characteristics of deep brain stimulation electrodes in vitro and in vivo. J Neural Eng 2009;6:046008.

29 Dorman MF, Smith LM, Dankowski K, McCandless G, Parkin JL: Long-term measures of electrode impedance and auditory thresholds for the Ineraid cochlear implant. J Speech Hear Res 1992;35:1126-1130.
30 Rouse AG, Stanslaski SR, Cong P, et al: A chronic generalized bi-directional brain-machine interface. J Neural Eng 2011;8:036018.

31 Farris S, Vitek J, Giroux ML: Deep brain stimulation hardware complications: the role of electrode impedance and current measurements. Mov Disord 2008;23:755-760.

32 Jaggi JL, Baltuch GH: Deep brain stimulation inactivity can produce unexpected high electrode impedances when reactivated, leading to a false conclusion of wire fracture. Stereotact Funct Neurosurg 2005;83:187-189.

33 Kim J, Richner TJ, Thongpang S, et al: Flexible thin film electrode arrays for minimally-invasive neurological monitoring. Conf Proc IEEE Eng Med Biol Soc 2009;2009:5506-5509.

34 Rosin B, Slovik M, Mitelman R, et al: Closedloop deep brain stimulation is superior in ameliorating parkinsonism. Neuron 2011;72: 370-384.

35 Neuburger J, Lenarz T, Lesinski-Schiedat A, Buchner A: Spontaneous increases in impedance following cochlear implantation: suspected causes and management. Int J Audiol 2009;48:233-239.

36 Ray A, Chan LL, Gonzalez A, Humayun MS, Weiland JD: Impedance as a method to sense proximity at the electrode-retina interface. IEEE Trans Neural Syst Rehabil Eng 2011;19: 696-699.

37 Schomberg D, Olson JK: Immune responses of microglia in the spinal cord: contribution to pain states. Exp Neurol 2012;234:262-270.

38 Fisher R, Salanova V, Witt T, et al: Electrical stimulation of the anterior nucleus of thalamus for treatment of refractory epilepsy. Epilepsia 2010;51:899-908.

39 Schaller K, Cabrilo I: Anterior temporal lobectomy. Acta Neurochir 2016;158:161-166. 